\title{
Feature Extraction and Classification for Insect Footprint Recognition
}

\author{
Bok-Suk Shin ${ }^{1}$, James Russell ${ }^{2,3}$, and Reinhard Klette ${ }^{1}$ \\ 1 Department of Computer Science, The University of Auckland \\ b.shin@auckland.ac.nz \\ 2 School of Biological Sciences, The University of Auckland \\ 3 Department of Statistics, The University of Auckland \\ Private Bag 92019, Auckland, New Zealand
}

\begin{abstract}
We propose a method to extract and classify insect footprints for the purpose of recognition. Our four-level procedural feature extraction model is defined as follows: First, images produce new data via the trace transform. Second, for reducing the dimensionality of the produced data, we apply some mathematical conversions. Third, dimensionalityreduced data are converted into frequency components. Finally, characteristic signals with significant components of representative values are created by excluding insignificant factors such as those related to noise. For classification, based on uncertain features, we propose a decision method defined by fuzzy weights and a fuzzy weighted mean. The proposed fuzzy weight decision method estimates weights according to degrees of contribution. Weights are assigned by ranking the degree of a feature's contribution. We present experimental results of classification by using the proposed method on scanned insect footprints. Experiments show that the proposed method is suitable for noisy footprints with irregular directions, or symmetrical patterns in the extracted segments.
\end{abstract}

\section{Introduction}

A method for monitoring small animals is defined by the use of tracking tunnels 1. Tracks are collected on white tracking cards having a pre-inked middle part. Animals, attracted by lures, walk through the tracking tunnel and leave their footprints on the tracking card. In environmental surveillance, footprint analysis helps for solving monitoring tasks, for example by verifying the presence of some rodents or insects, or for more detailed ecological or biological studies as supported by those footprints [23]. The identification requires that individual footprints are extracted, and then clustered into meaningful track patterns. In general it is even difficult for experts to analyse, extract, or classify footprints.

A track of footprints typically has a symmetrical structure (left and right feet centring on the body, see Fig. 1), but are translated or rotated into irregular directions. Thus we can expect to extract similar prints for the left side and right side, but affected by affine transformations. Binarization of collected images needs to be adaptive to deal with varying intensities, but the binarization process will also contribute to uncertainties. The applied classification method can only use the described incomplete information for footprint identification. 


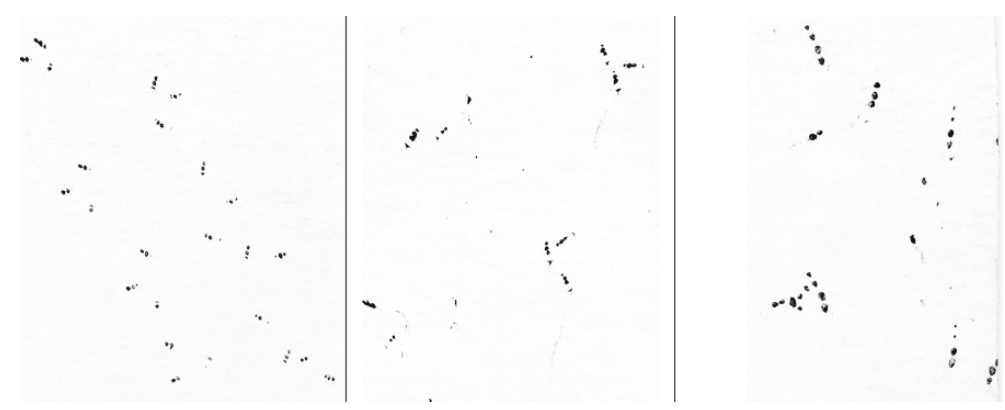

Fig. 1. Footprints in areas of tracking cards; left to right: black cockroach (Platyzosteria sp.; class A in our experiments), native bush cockroach (Celatoblatta sp.; class B), and ground weta (Hemiandrus sp.; class C)

In order to overcome these difficulties, we propose a new feature extraction and classification method. For extracting features, we use a 4-level procedural model that is suitable for footprints having characteristics invariant to affine transformations. For pattern recognition and classification, we propose a fuzzy weight decision, where weights are based on degrees of contributions, considering the possibilities of image degradations.

\section{A 4-Level Procedural Feature Extraction Model}

We propose a 4-level procedural feature extraction model. This model is suitable for extracting features for noisy footprints of insects, small mammals and so forth, having irregular directions, a symmetrical pattern structure, and a few singularities in extracted segments [4]. The 4-level procedural feature extraction method (4-level FEM) is summarized as follows. First, images produce new 2D data (the trace matrix) through the trace transform [5]. Second, the trace matrix is processed for reducing the dimensionality of the data by several mathematical conversions. Third, dimensionality-reduced data are converted into frequency components. Finally, characteristic signals (CS-feature) with significant components of representative values are created by excluding insignificant factors.

At Level 1, a footprint segment produces new data (basic features) through the trace transform. The trace transform can create feature values of an input image that are invariant to rotation, translation, and even reflection of the input image. Thus it is appropriate to extract feature values from various shapes of animal footprint segments, even if deformed by rotation, translation or reflection. The trace transform, as used in this paper, is as follows:

Let $F$ denote an image. We position a $2 \mathrm{D}$ coordinate system in the image (e.g., origin at the centre) and represent features of image $F$ with respect to straight lines $l(\theta, p)$ (called trace lines), where $\theta$ is the angle with the horizontal axis, and $p$ the distance to the origin. See Fig. 2, left. We have $l=\{(x, y)$ : $x \cos \theta+y \sin \theta=p\}$. Assuming that we have a trace function $T$, we finally have the trace transform defined by $T_{F}(\theta, p)$. The position of the origin is not 

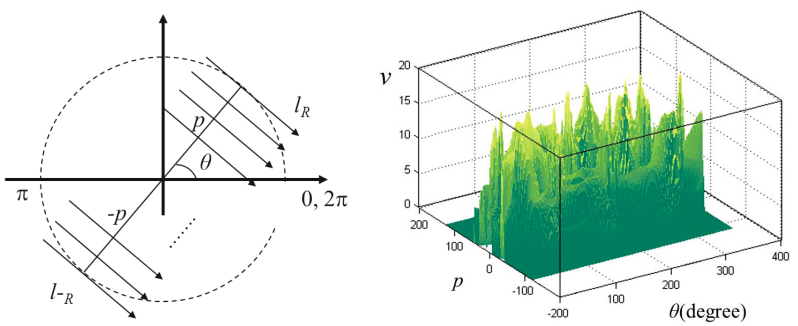

Fig. 2. Left: parameters $\theta$ (angle) and $p$ (distance) define a straight line $l_{R}$. Right: a $3 \mathrm{D}$ visualization of a trace matrix, with $V$ (value) as the third axis.

important for the qualitative analysis of the resulting trace matrix $\mathbf{T}_{F}$. See Fig. 2, right, with $v=T_{F}(\theta, p)$. We do not use subscript $F$ if not needed.

Following [5], if the original image rotates then its trace matrix shifts along the horizontal axis; if the original image translates by some vector then its trace matrix also undergoes defined changes: columns remain unchanged and stay in their places but may shift up or down. A shift vector specifies numbers $a$ and $b$ such that a column with coordinate $\theta_{i}$ shifts vertically to $a \cos \left(\theta_{i}-b\right)$. Because of the characteristics, feature values extracted from an input image by the trace transform are always invariant to translation and rotation. Altogether, Level 1 is defined by the following:

1-1. The trace transform is determined by the trace function $T$.

1-2. The trace-line $l$ is decided using the distance $p$ from the origin to $l$, and the angle $\theta$, the trace-line $l$ is represented by the formula $l=\{(x, y)$ : $x \cos \theta+y \sin \theta=p\}$.

1-3. A trace matrix $\mathbf{T}_{F}$ is generated by the trace transform; matrix $\mathbf{T}_{F}$ can be visualized in $3 \mathrm{D}$ space using axes $\theta, p$, and $v$; the range of $\theta$ is $[0,2 \pi]$, and the range of $p$ is $\left[p_{\min }, p_{\max }\right]$.

1-4. Two different trace functions $T(\theta, p)=T_{F}(\theta, p)$ are defined as follows:

$$
T_{1}(\theta, p)=\int_{k \in l} \eta(k) \mathrm{d} k \quad \text { and } \quad T_{2}(\theta, p)=\int_{k \in l} t^{2} \eta(k) \mathrm{d} k
$$

where $k$ is a pixel location in image $F$, function $\eta$ outputs a value at pixel $k$ along trace line $l, t=\eta(k)-c$, and $c$ is the mean of all $\eta(k)$ along trace line $l$ in image $F$.

At Level 2, the produced data are processed for reducing the dimensionality of the data by several mathematical conversions. Level 2 is defined by the following:

2-1. A diametric signal $\rho$ is generated by a diametric function $P$; the range of the diametric signal $\rho$ is for all $\theta$ in $[0,2 \pi]$.

2-2. Four different diametric functions $P(\theta)$, for $t=\eta(h)-\operatorname{median}\{\eta(h)\}$ :

$$
\begin{aligned}
& P_{1}(\theta)=\int_{p} \eta(h) \mathrm{d} h \quad \text { and } \quad P_{2}(\theta)=\int_{p} t^{2} \eta(h) \mathrm{d} k \\
& P_{3}(\theta)=\max _{p}\{\eta(h)\} \quad \text { and } \quad P_{4}(\theta)=\operatorname{var}_{p}\{\eta(h)\}
\end{aligned}
$$

where $h=(\theta, p)$, and function $\eta$ outputs a value at point $h$. Figure 3 , left, shows $\rho$ signal generated by Level 2 . 

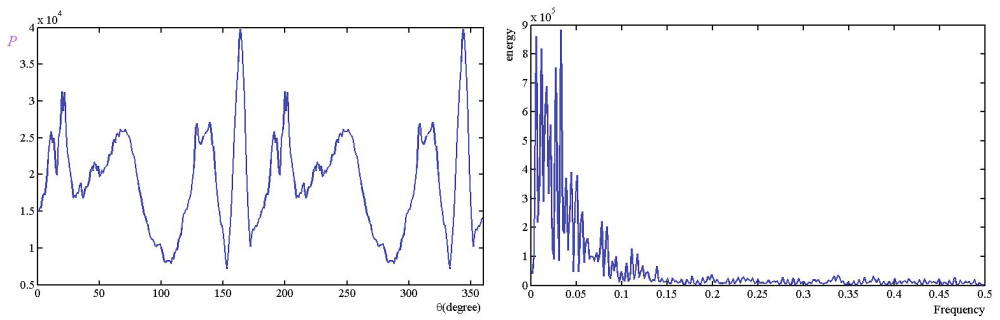

Fig. 3. Left: $\rho$ signal through function $P$. Right: frequency signal $\phi$

At Level 3, dimensionality-reduced data are converted into frequency components. We extract frequency components from the data set to get the range of signal waves regardless of the data translation by the Fourier transform. A frequency transform divides a signal into high or low frequency parts in order to adjust them, to analyse a cycle of a specific signal wave, or to obtain the range of a signal wave. Level 3 is defined by the following:

3-1. The signal $\phi$ is acquired by the circus function $\Phi$ using parameter $\theta$; the range of signal $\phi$ is $[0,2 \pi]$.

3-2. The circus function $\Phi$ is defined by $\phi=F F T(\rho)$.

Figure 3 , right, shows signal $\phi$ generated by Level 3.

Finally, at Level 4, characteristic signals with significant components of representative values are created by excluding insignificant factors such as noise. A characteristic function $\zeta$ is a filtering method that chooses $n$ high energy elements on the frequency axis. This ensures comparison of strong harmonic signals, excluding meaningless features in the frequency components. A strong characteristic signal is a way to represent a spectrum's shape. The characteristic function $\zeta$ defines values $\Lambda=\zeta(\phi)$ CS-features are extracted by the 4-level FEM, which is the feature used in the final pattern classification step.

\section{A Fuzzy Network for Classification}

In the case of scanned tracks of footprints, insect footprints are 'tiny' scattered spots and form delicate patterns. The intensity of a footprint mostly depends on ink quantity on a pad. Those images of footprints have frequently not only a few incomplete spots that are not filled with black ink, but also sliding (smearing) footprints, missing toe footprints, or overlapped footprints because of movements. In addition, footprint segments show lots of noise not yet removed in the process of segmentation. Fuzzy theory is used for appropriate evaluation of, or decisions on, uncertain data [6]. Membership functions of fuzzy sets can take on any shape, such as triangular, trapezoidal, exponential, Gaussian, or cosine. In this paper, we generate membership values by using a triangular membership function [7]. We use a fuzzy network consisting of 6 layers.

Layer 1. Let $f$ be a set of input features for one of the classes $i=1$ to $k$. Each input set $f$ defines a fuzzy function, represented by a data array of size $1 \times e$, where $e$ is the number of features. 
Layer 2. Membership values are determined by a triangular membership function in the unit interval $[0,1]$, with mean $m(f)$ at maximum membership value 1 , and interval borders $v(f)_{\min }$ and $v(f)_{\max }$ at minimum membership value 0 . The triangular membership function is represented as follows:

$$
u_{j i}\left(f_{i}\right)=\left\{\begin{array}{l}
\frac{1}{\left(m_{i}-v_{i_{\min }}\right)}\left(f_{i}-m_{i}\right)+1 \\
-\frac{1}{\left(v_{i_{\max }}-m_{i}\right)}\left(f_{i}-m_{i}\right)+1
\end{array}\right.
$$

Layer 3. Weights are computed by fuzzy weighted mean.

Layer 4. The membership value is multiplied with the weight and used as input to Layer 5: $U_{i}(f)=u_{i}(f) \cdot w_{j}$.

Layer 5 . The output of Layer 5 equals $\hat{h}_{i}=\sum_{j=1}^{e}\left(U_{j}(f)\right)$.

Layer 6 . This layer finds the highest membership among all outputs of Layer 5 . The class $i$ having the highest membership value $\hat{h}_{i}$ is the final classification result, for $i=1,2, \ldots, k$.

\section{Fuzzy Weights Decisions}

We propose a decision method for fuzzy weights by estimating the weight according to degrees of contribution. The weights are assigned by ranking the degrees of the contribution. The degrees of contribution are decided by parameters comparing the classes. If one of the feature values defines a distinct difference for deciding classification, a high weight is assigned to this feature. Calculated weights determine membership values by fuzzy membership functions. Objects are classified into that class which has a superior value.

We assign to each feature its weight according to parameters such as its variance, gap, or overlap with other classes. Decided weights are assigned by the fuzzy weighted mean method. The fuzzy weighted mean equation is as follows:

$$
u_{j i}\left(f_{i}\right)=\sum_{j=1}^{e}\left(u_{j i}(f) \cdot N_{w_{j}}\right)=\sum_{j=1}^{e}\left(U_{j i}(f)\right)
$$

Membership function values are real numbers. A triangular graph is used to define how each point in the input space is labelled by a membership value between 0 and 1. Such a triangular membership graph is defined by classes which are separated by straight line segments. A gap between two straight lines defines a gradient; the gap is defined by variance. Therefore, membership graphs have gradient and variance data assigned. Such graphs may be non-disjoint for different classes. We use mean and variance in non-empty intersections of membership graphs for deciding about weights. Consider classes $A$ and $B$ with centroids $A_{c}$ and $B_{c}$. For the distance between class $A$ and class $B$ we take the distance between $A_{c}$ and $B_{c}$.

We decide about weights based on a degree of contribution. In the proposed method we use parameters such as mean and variance, the ratio of variance in a class, and the distance between two classes. See Fig. 4 


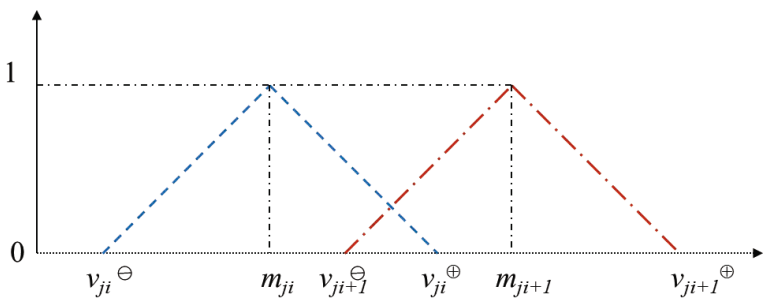

Fig. 4. Variables for adjusting weights

Let $C$ be a class. Let $V$ be the variance of $C$ and $m$ its mean. $V(m)$ is the variance of means of other classes, $g^{n}$ is the distance of separation among other classes, and $g$ is overlapping among other classes. The weight $w_{j i}$ is defined as follows:

$$
\begin{aligned}
w_{i} & =-\frac{\sum g_{i}^{n}+1}{\sum g_{j i}} \times\left(V\left(m_{j i}\right)+1\right) \\
g_{j i}^{n} & =-\frac{d_{i j}}{V\left(m_{j i}\right)} \text { if } d_{i j}>0 \quad \text { and } g_{j i}=-\frac{d_{i j}}{V\left(m_{j i}\right)} \text { if } d_{i j}<0
\end{aligned}
$$

where, if $\sum g_{j i}=0$ then use $\sum g_{j i}=1$, and $d_{j i}=v_{j i+1}^{\ominus}-v_{j i}^{\oplus}$ for $i=a$ is the number of classes, $j=a$ the number of features, and $V\left(m_{j i}\right)=\left\|v_{j i}^{\oplus}-v_{j i}^{\ominus}\right\|$. Let $w_{j}^{\Omega}=\left(w_{j}-\left(w_{j}\right)^{M i n}\right) /\left(\left(w_{j}\right)^{M a x}-\left(w_{j}\right)^{M i n}\right)$. Let $N_{w_{i}}$ be the normalized weight $w_{j}^{\Omega}$ such that $\sum_{j=1}^{e} N_{w_{j}}=1$.

\section{$5 \quad$ Footprint Classification and Experiments}

In order to classify footprints, we need to extract different singular features from segments and to compose patterns by combining feature sets. It is possible to classify footprints based on uniquely identified patterns. We can compare using the variance of features if they are distinct (i.e. their 'character' is reflected in their distribution). Therefore, we generate CS-features by using the 4-level FEM. For footprint classification, we consider gradually 2-step classifications to increase the reliability of evaluation. The 4-level FEM is able to generate $n$ kinds of features. In

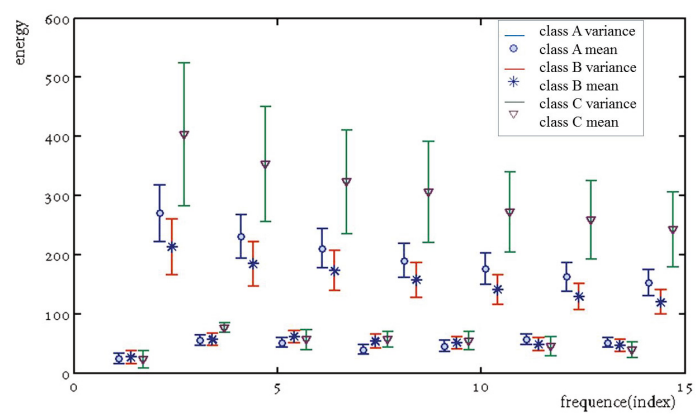

Fig. 5. Distribution of extracted CS-features 


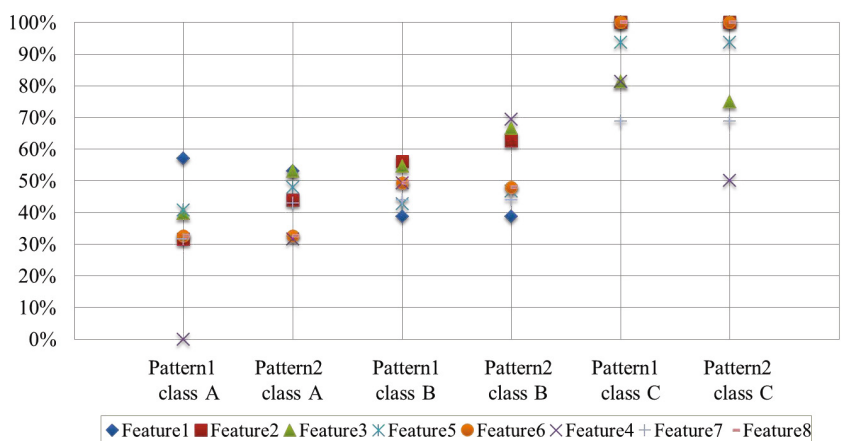

Fig. 6. Recognition results by the first classification step

other words, we can obtain $n$ membership values as well as $n$ results of classification decided by $n$ membership values. We reclassify using $n$ results of classification.

In a primary classification, classification happens by the proposed fuzzy weighted-mean model; $n$ results of classification are made, which show different results or the same result of classification, $h$ is the result of classification, and $h^{*}$ is the highest value of the class. In a secondary classification, in the case of different results, we need to decide a final result to determine which class. We use a majority voting approach. There are $n$ values $h^{*}$ after primary classification. We choose the classification based on voting: let $h^{*}$ be the set $\Upsilon$ defined as $\left\{h_{1}^{*}, h_{2}^{*}, \cdots, h_{n}^{*}\right\}$; the final result is $\hat{\Upsilon}=$ vote $[\Upsilon]$.

In our experiments, we extracted CS-features from improved segments by using the 4-level FEM, and we generated patterns by combining each of the eight kinds of features. We used footprints of three insect species: black cockroach (class $A)$, native bush cockroach (class $B$ ), and ground weta (class $C$ ). Figure 1 shows three kinds of footprints on a tracking card. The number of samples used was class $A=102$, class $B=61$, and class $C=16$. Figure 5 shows the distribution of CS-features

Table 1. Recognition results by the second classification step

\begin{tabular}{ccccccc}
\hline & & \multicolumn{5}{c}{ Voting result } \\
\cline { 5 - 6 } Insect Class & Card No. & Class & Class B & Class C & Recognition \\
\cline { 5 - 6 } Class A & $\# 1$ & 9 & 5 & 1 & True \\
& $\# 2$ & & 4 & 7 & 4 & False \\
& $\# 3$ & 8 & 5 & 4 & True \\
& $\# 4$ & 8 & 7 & - & True \\
& $\# 5$ & 8 & 5 & 4 & True \\
& $\# 6$ & 5 & 2 & 5 & - \\
& $\# 7$ & 5 & 6 & - & False \\
\hline Class B & $\# 1$ & 3 & 5 & 2 & True \\
& $\# 2$ & 5 & 1 & 1 & False \\
& $\# 3$ & 3 & 4 & 3 & True \\
& $\# 4$ & 2 & 9 & - & True \\
& $\# 5$ & 1 & 6 & - & True \\
& $\# 6$ & - & 7 & 1 & True \\
& $\# 7$ & 1 & 7 & - & True \\
\hline Class C & $\# 1$ & - & - & 4 & True \\
& $\# 2$ & - & - & 4 & True \\
& $\# 3$ & - & - & 3 & True \\
& $\# 4$ & - & - & 5 & True \\
\hline
\end{tabular}


extracted from segments of footprints, which are extracted by 4-level FEM. Each class has mean and variance as defined above. In our experiments, we performed gradually a 2-step classification. The first step is recognition depending on fuzzy membership values of segments (see Fig. [6). This shows recognition results by the first classification. For recognition evaluation, we used eight kinds of features, defining two types of patterns. See the case of class $C$ in Fig.66. Feature 4 has a recognition rate of $69 \%$, Feature 6 has a recognition rate of $100 \%$ when using the first pattern (see Tab.11). This table shows recognition results by the second classification step (i.e. voting with the results after the first classification). For example, regarding the class $A$ insect and Card \#3, 8 segments of footprints indicate that they are class $A, 5$ segments of footprints say that they are class $B$, and 4 segments of footprints say that they are class $C$. Therefore, the majority is class $A$. On seven cards we had class $A$ or class $B$, and on four cards class $C$.

\section{Conclusions}

This paper proposed a feature extraction method and a classification method for the purpose of diverse small species recognition by using their footprint images. We proposed a 4-level FEM. This model is suitable for extracting features of species with irregular directions, symmetrical structure patterns, and noisy prints with a few singularities in the extracted segments. For classification, based on uncertain features, we proposed a decision method defined by fuzzy weights and a fuzzy weighted mean. We presented experimental results of classification by using the proposed method on scanned insect footprints of three species.

Acknowledgement. The data used in this paper were provided by the insect track recognition project at The University of Auckland. The project was initiated in 2003, in collaboration with Connovation Ltd., Auckland.

\section{References}

1. Blackwell, G.L., Potter, M.A., McLennan, J.A.: Rodent density indices from tracking tunnels, snap-traps and fenn traps: do they tell the same story? New Zealand J. Ecology 26, 43-51 (2002)

2. Russell, J.C., Hasler, N., Klette, R., Rosenhahn, B.: Automatic track recognition of footprints for identifying cryptic species. Ecology 90, 2007-2013 (2009)

3. Whisson, D.A., Engeman, R.M., Collins, K.: Developing relative abundance techniques (RATs) for monitoring rodent population. Wildlife Research 32, 239-244 (2005)

4. Shin, B.-S., Cha, E.-Y., Kim, K.-B., Cho, K.: -W., Klette, R., and Woo, Y. W., Effective feature extraction by trace transform for insect footprint recognition. J. Computational Theoretical Nanoscience 7, 868-875 (2010)

5. Petrou, M., Kadyrov, A.: Affine invariant features from the trace transform. IEEE Trans. Pattern Analysis Machine Intelligence 26, 30-44 (2004)

6. Jin, Y.: Fuzzy modelling of high-dimensional systems complexity reduction and interpretability improvement. IEEE Trans. Fuzzy Systems 8, 212-221 (2000)

7. Olunloyo, V.O.S., Ajofoyinbo, A.M.: On the systematic computation of membership functions of union and intersection of triangular fuzzy. European J. Scientific Research 41, 135-149 (2010) 